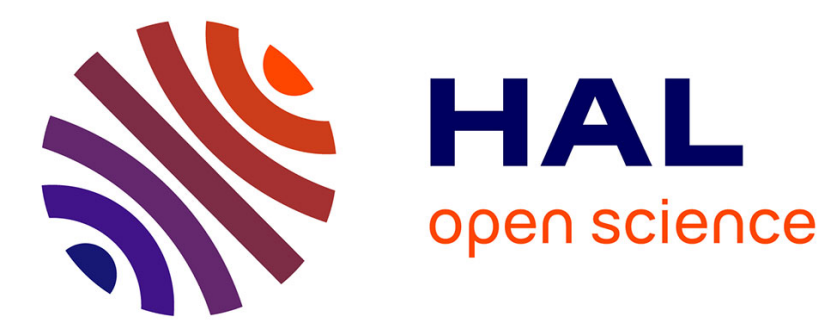

\title{
Automatic evaluation of form errors in high-density acquired surfaces
}

Luca Di Angelo, Paolo Di Stefano, Anna Eva Morabito

\section{To cite this version:}

Luca Di Angelo, Paolo Di Stefano, Anna Eva Morabito. Automatic evaluation of form errors in high-density acquired surfaces. International Journal of Production Research, 2010, pp.1. 10.1080/00207541003657370 . hal-00588675

\section{HAL Id: hal-00588675 https://hal.science/hal-00588675}

Submitted on 26 Apr 2011

HAL is a multi-disciplinary open access archive for the deposit and dissemination of scientific research documents, whether they are published or not. The documents may come from teaching and research institutions in France or abroad, or from public or private research centers.
L'archive ouverte pluridisciplinaire HAL, est destinée au dépôt et à la diffusion de documents scientifiques de niveau recherche, publiés ou non, émanant des établissements d'enseignement et de recherche français ou étrangers, des laboratoires publics ou privés. 


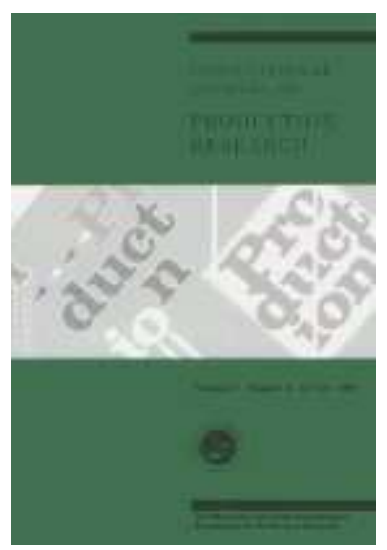

\section{Automatic evaluation of form errors in high-density acquired surfaces}

\begin{tabular}{|r|l|}
\hline Journal: & International Journal of Production Research \\
\hline Manuscript ID: & TPRS-2009-IJPR-0660.R2 \\
\hline Manuscript Type: & Original Manuscript \\
\hline Author: & 17-Jan-2010 \\
\hline Complete List of Authors: & $\begin{array}{l}\text { Di Angelo, Luca; Universita' degli Studi di L'Aquila, Department of } \\
\text { Mechanical Engineering } \\
\text { Di Stefano, Paolo; Universita' degli Studi di L'Aquila, Department of } \\
\text { Mechanical Engineering } \\
\text { Morabito, Anna Eva; University of Lecce, Department of "Ingegneria } \\
\text { dell'Innovazione" }\end{array}$ \\
\hline Keywords: & TOLERANCING, INSPECTION \\
\hline Keywords (user): & Geometric Product Specification, Form Errors \\
\hline &
\end{tabular}

\section{S) ScholaroNE \\ Manuscript Central}




\title{
Automatic evaluation of form errors in high-density acquired surfaces
}

\author{
L. DI ANGELO $\left({ }^{1}\right)$, P. DI STEFANO $\left({ }^{1}\right)$, A. E. MORABITO $\left({ }^{2}\right)$ \\ ( $\left.{ }^{1}\right)$ Department of Industrial Engineering, University of L'Aquila, \\ Loc. Monteluco di Roio, 67030 L'Aquila, Italy \\ (2) Department of "Ingegneria dell'Innovazione”, University of Lecce, \\ Via per Monteroni, 73100 Lecce, Italy
}

(Received; final version received)

\begin{abstract}
In this paper the authors present an original methodology aiming at the automation of the geometric inspection, starting from a high-density acquired surface. The concept of intrinsic nominal reference is herein introduced in order to evaluate geometric errors. Starting from these concepts, a new specification language, which is based on recognizable geometric entities, is defined. This work also proposes some surface differential properties, such as the intrinsic nominal references, from which new categories of form errors can be introduced. Well-defined rules are then necessary for the unambiguous identification of these intrinsic nominal references. These rules are an integral part of the tolerance specification.

This new approach requires that a recognition process be performed on the acquired model so as to automatically identify the already-mentioned intrinsic nominal references. The assessable errors refer to recognizable geometric entities and their evaluation leaves the nominal reference specification aside since they can be intrinsically associated with a recognized geometric shape. Tolerance specification is defined based on the error categories which can be automatically evaluated and which are an integral part of the specification language.
\end{abstract}

Keywords: GPS tolerancing, automated inspection, form error evaluation.

\section{Introduction}

The verification process is the manufacturing step at which a metrologist determines whether the real surface of a workpiece conforms to the range of permissible deviations that have been specified during the design process. These specifications express the functional requirements that have to be satisfied so that the manufactured parts work as designed. In the last few years, geometric inspection has become critical due, on the one hand, to the increase of geometrical complexity and product variety, and on the other hand to the ever-growing demands for geometrical accuracy. Consequently, a reliable, effective and automated inspection system will definitely improve the industrial manufacturing responsiveness and increase quality and product competition.

In order to harmonize and fill in the gaps of what has already been standardized in tolerancing and metrology by the ISO, the technical committee ISO/TC 213 has recently proposed a language, supported by mathematical principles, which is referred to as Geometrical Product Specification (GPS).

The measuring equipments, with their specific way to acquire the geometric properties of the object under verification, affect error evaluation and specification; GPS standards include this concept. With the advent of CMM (Coordinate Measuring Machines) and high-resolution optical digitizers, alternative approaches for 
dimensional and geometrical error definition and evaluation can be identified. In particular, nowadays, the approach to be used cannot ignore the fact that a large amount of point data can be extracted from the workpiece itself, by means of optical 3D scanners characterized by an increasingly high accuracy. The measurement carried out by these devices is not a set of single measures (as it is the case when using a CMM device); rather, it consists in a 3D acquisition of the real object. The acquired points identify the surfaces of the object so it is possible to recognize from them the geometric properties from which to detect references for error evaluation. The acquired surface can be compared to the recognized nominal entities which assume the role of virtual references because of their mathematical definition. For this reason, new categories of nominal reference can be conceived which may improve on the signs in the traditional language for tolerance specification. Error evaluation always requires the knowledge of the corresponding nominal reference. In this work nominal references are classified into two main categories: explicit and intrinsic. The explicit reference can be given by a specification, also by using a CAD model (Prieto et al. 2002, Gao et al. 2006). Intrinsic references are nominal entities that can be recognized in the scanned object, independently of the tolerance specification.

This paper then presents an original methodology aiming at the automation of the inspection, starting from a high-density acquired surface. This new approach requires that a recognition process be performed on the acquired model so as to automatically identify the intrinsic nominal references. The identification of intrinsic nominal references avoids the registration process between CAD model and data points. What is here carried out is an original classification of the form error categories for the recognized surfaces based on the above-mentioned concepts. Specific form tolerances are defined for any recognizable surface. These categories, together with the traditional ones, have been implemented in an original software and tested in real cases. An example is hereafter presented.

\section{Related works}

Recent efforts have been made to develop methods which apply non-contact digitizing techniques to the geometric inspection. A great number of the methodologies proposed in literature require the knowledge of the CAD model of the workpiece under inspection, which provides the nominal references in the form of the analytical surface describing the geometric model (Prieto et al. 2002, Li and Gu 2004, $\mathrm{Li}$ and $\mathrm{Gu}$ 2005, $\mathrm{Li}$ and $\mathrm{Gu}$ 2005, Gao et al. 2006). Since tolerance specifications usually refer to some features of the workpiece, the mapping between one surface (or feature) of the CAD model and the corresponding scanned point sub - cloud has to be performed. Tolerance specifications can be either included in the CAD model as textual information, or interactively defined by the user. Nowadays no standard language able to specify tolerances and suited to automatic verification has yet been defined. Prieto et al. in (Prieto et al. 2002) propose and implement a methodology for the automated inspection of manufactured parts. This methodology is able to verify both dimensional and geometric tolerances. Firstly, the high-density point cloud is registered with the corresponding CAD model of the workpiece by means of the Iterative Closest Point (ICP) algorithm. The 3D point cloud is then segmented by associating the points matching the same local geometric properties with the nearest CAD surface. Since the registration process minimizes the sum of the squared distances between the point cloud and the CAD model, the authors state that the 
distances, between a generic CAD surface and the corresponding sub - cloud, will have a Gaussian distribution. The specified errors are estimated based on the average value and the standard deviation of the point location distribution. Particularly, the form error is evaluated as four times the standard deviation of the distances between the points and the analogous CAD surface which approximates them. A similar approach is proposed by $\mathrm{Li}$ and $\mathrm{Gu}$ ( $\mathrm{Li}$ and $\mathrm{Gu} 2004, \mathrm{Li}$ and $\mathrm{Gu} 2005, \mathrm{Li}$ and $\mathrm{Gu}$ 2005). Gao in (Gao et al. 2006) develops an automated geometric inspection system within a commercial RE software. The authors define a Nominal Inspection Frame (NIF) for a CAD model or a reference digitized model (Master Model) in which every dimensional and geometrical tolerance specification may be defined and interactively specified by the user. A similar approach is proposed by Campana et al (Campana et al. 2006), which automatically performs the mapping between the tolerance specification in the CAD model and the corresponding acquired sub - cloud.

All these approaches evaluate the form errors in the same way as the "profile tolerance of a surface" does (ISO 1101: 2004) (the tolerance zone is limited by two CAD surfaces placed up and down the CAD reference surface at a distance $t / 2$ from it), without taking into account specific geometric properties of the surface (for example, axially - symmetric surface, extruded surface, etc.). Some specific properties of the surface can actually play an important functional role in the object. For this purpose, ISO 1101 and ASME 14.5Y consider form tolerances, such as straightness, circularity, etc., which can be applied to derived or extracted features from the surface (axis, planar section of the surface, etc.). The approaches presented in literature, which use a CAD model as an analytical reference, show limitations if compared to the traditional approach to tolerance inspection. In other words, the specification "language" based on these methods is generally characterized by fewer terms than in the traditional approaches of tolerance specification. Furthermore, these approaches do not take advantage of the specific way to inspect the real object and the numerical devices that can be used to evaluate the acquired point cloud. Based on these specific characteristics, new categories of form error can be introduced in accordance with the duality principle reported in GPS standards (Nielsen 2006). Finally, another drawback of the tolerance inspection methods based on CAD systems is the lack of standards for geometric data exchange.

\section{Automatic error evaluation}

The methodology proposed in this paper is suited to automatically evaluate the form errors of high-density acquired surfaces. The flowchart of the methodology is shown in figure 1 . It can be decomposed into the following four steps:

(1) Non-ideal feature recognition;

(2) Nominal reference association;

(3) Error evaluation;

(4) Form tolerance evaluation.

During the first phase, the GPS feature operation, called partition, is carried out. In the GPS language this operation aims at "obtaining, from the non-ideal surface model (skin model) or the real surface, the non-ideal features corresponding to the nominal features" (ISO/TS 17450-1: 2005).

In this work the non-ideal feature is defined as a set of adjacent mesh vertices that are recognized as smooth and of the same type (flat, umbilical, ruled and generic). The concept of non-ideal feature then involves the concept of point 
smoothness. In a continuous surface a point is smooth if its neighbourhood can be represented by a smooth function. In other words, in a non - smooth point the differential geometrical properties cannot be defined. Typically, non -smooth points are those pertaining to the edges of the geometrical model where two different surfaces meet. The concept of smooth point can be extended from a point of a continuous surface to a vertex of a tessellated surface. In a tessellated surface the point smoothness property must be recognized, by using some criteria (Di Angelo and Di Stefano 2009).

Error evaluation always requires the knowledge of a nominal reference from which the error can be measured. The nominal reference can either be given explicitly (for example, by a GPS specification or a CAD model) or can be implicitly or intrinsically present in the acquired object. In the related literature several researchers have used a CAD model as a nominal reference (Prieto et al. 2002, Gao et al. 2006). In this work the nominal references are recognized as intrinsic references from the acquired workpiece. The categories of valuable errors can be deduced from these references. Error evaluation involves the quantitative identification of the reference element from which the deviation can be evaluated. In figure 1 this phase is referred to as Intrinsic Nominal Reference association. For this purpose, some specific rules, referred to as association rules, need to be introduced. They play an important role in quantitatively identifying the references; in fact, different rules can produce different estimations of the references. These rules then assume the role of identifiers of nominal references just as the reference surfaces of a gauge do in the traditional approach to error measurement.

In the next phase, and for each identified intrinsic reference, the deviations from the corresponding associated non-ideal feature are evaluated and assumed as form errors.

The procedure ends with the comparison between the estimated form errors and the GPS specifications. Thus, from all the errors associated with all the intrinsic references identified, some are extracted based on the tolerance specifications.

\section{Non-ideal feature recognition}

To carry out a reliable recognition of the non-ideal feature types it is necessary to segment the acquired surface into regular continuous patches. Segmentation is a complex procedure that makes it possible to capture high-level geometric information that is essential for the following Intrinsic Nominal Reference association. The non-ideal feature recognition is carried out based on the procedure outlined in figure 2, where two main segmentation levels are identified.

The first level aims at identifying regular surfaces or, equivalently, at detecting surface discontinuities. The second level is intended for recognizing the geometric shape by investigating some intrinsic local and global differential properties of the tessellated model. The evaluation of these differential properties is a complex task since the triangulated model represents only a discrete approximation of the smooth geometry. In literature, two are the main approaches used for data segmentation; namely, the edge-based, the region-based methods (Liu and Xiong 2008). In order to overcome the lack of robustness, non-uniqueness or complexity deriving from the use of one of these two strategies, some hybrid approaches, based on edge-based and region-based methods, have been developed. This work makes use 


\section{Intrinsic nominal reference}

In order to perform an automatic error evaluation, we introduce the original concept of Intrinsic Nominal Reference. The Intrinsic Nominal Reference is associated with the concept of recognisability. There exists an Intrinsic Nominal Reference when a property is recognized as common to a set of adjacent points of the point cloud of the acquired object. For example, planarity is an intrinsic property since it can be recognized in a set of points that are evaluated as sufficiently close to a plane surface. In this paper three different types of intrinsic nominal references are being considered:

- Intrinsic Shape Reference (ISR);

- Intrinsic Derived Reference (IDR);

- Intrinsic Local Reference (ILR).

Depending on the recognized non-ideal feature type, one or more Intrinsic Nominal References can be identified. Table 1 shows the intrinsic references related to each recognized non-ideal feature.

Table 1. Non-ideal feature types and the related intrinsic references

\begin{tabular}{l|c|c|c|}
\multirow{2}{*}{ Non-ideal feature type } & \multicolumn{2}{|c|}{ Intrinsic Reference Types } \\
\cline { 2 - 4 } plane & Analytical shape & IDR & ILR \\
\hline sphere & Analytical shape & - & Surface regularity \\
\hline \multirow{2}{*}{ cylinder } & Analytical shape & Ideal circumferential line & $\begin{array}{c}\text { Surface regularity } \\
\text { Profile regularity } \\
\text { Ruledness }\end{array}$ \\
\hline \multirow{2}{*}{ cone } & Analytical shape & Ideal circumferential line & $\begin{array}{c}\text { Surface regularity } \\
\text { Profile regularity } \\
\text { Ruledness }\end{array}$ \\
\hline generic extruded & & & $\begin{array}{c}\text { Surface regularity } \\
\text { Profile regularity } \\
\text { Ruledness }\end{array}$ \\
\hline generic cone & & - & $\begin{array}{c}\text { Surface regularity } \\
\text { Ruledness }\end{array}$ \\
\hline generic axially- & & - & $\begin{array}{c}\text { Surface regularity } \\
\text { Pymmetric }\end{array}$ \\
\hline free form & - & Axis & Surface regularity \\
\hline
\end{tabular}




\subsection{Intrinsic Shape Reference}

The Intrinsic Shape Reference is recognized in those points of the acquired workpiece which can be considered as lying on an analytical surface (for example, plane, sphere, cylinder, cone, torus, etc.). The recognition of this type of reference requires the identification of an analytical surface, which approximates the points belonging to the tessellated surface. The concept of non-ideal feature is more general than that of Intrinsic Shape Reference since it can also include the smooth free-form surfaces. For these surfaces it is not possible to identify an Intrinsic Shape Reference. This nominal reference can be provided by a CAD model, as it is the case in other approaches described in literature (Prieto et al. 2002, Gao et al. 2006). The Intrinsic Shape References are recognized by means of some rules which play a leading role in the definition of the domain of the recognizable intrinsic references and of the assessable errors.

\subsection{Intrinsic Derived Reference}

The Intrinsic Derived References are the analytically known geometric entities deriving from the recognition of some geometric properties. These entities are not physical and, for this reason, are not directly measurable from the surface, but can nevertheless be derived from the surface. In many cases these references are the situation features of the non-ideal feature (for example, the axis for a generic axiallysymmetric or helicoidal surface). In other cases they are special sections of the surface (for instance, the geometric shape of the directrix curve for a generic extruded surface, the generatrices of a ruled surface or the circular cross-section for a generic axially-symmetric surface).

\subsection{Intrinsic Local Reference}

The Intrinsic Local Reference is not considered in the current tolerancing standards. It deals with the uniformity of some intrinsic differential geometric properties, such as: regularity, curvature recurrences, and so on. These references do not pertain to the global analytical properties of a surface, but rather to properties which locally characterize it. In what follows, two Intrinsic Local References are introduced.

\section{Regularity}

One of the most important Intrinsic Local References is the regularity of the points pertaining to a continuous surface or, more properly, to a non-ideal feature. A continuous surface is regular if the following two conditions are satisfied (Do Carmo 1974):

(1) At each point, derivatives of any order exist in any direction(which are automatically continuous). A surface which meets this condition is referred to as differentiable surface;

(2) At each point, there exists a tangent plane (i.e. there are no singular points).

A regular surface is, roughly speaking, assimilable to a differentiable surface and any imperfection and/or error is associated with a deviation from it. A tessellated surface is a non-regular reconstruction of an acquired surface, which can be 
assimilated to a nominally regular surface. For this reason, surface regularity is not a characteristic of the acquired point cloud, but it should be recognized from the cloud as an intrinsic reference. The surface regularity measurement is useful for the evaluation of the surface imperfections. For example, surface imperfections, such as scratches, cracks and acquisition errors, can produce surface regularity errors. This kind of intrinsic reference can be recognized also for surfaces that are not analytical.

In the case of generic extruded or axially-symmetric surfaces it could also be useful to distinguish the geometrical property of the surface transverse section, which has a nominal analytical reference (straight line or circle), from the longitudinal section which, generally speaking, cannot be assimilated to an analytical reference. In order to evaluate this type of imperfections, the concept of profile regularity can be introduced. For instance, for a turned generic axially-symmetric surface, any surface imperfection, which is associated with an undesired circumferential groove caused by the tool vibration, will not give rise to axis straightness error or to circularity error; the imperfection will nonetheless be identified because of a loss of profile regularity.

\section{Ruledness}

Another Intrinsic Local Reference, introduced in this work, concerns ruled surfaces. This reference has to do with the confirmation of the ruled property of the acquired surface. It assumes an important role in generic ruled surfaces for which a reference of the type ISR cannot be identified. For this type of surfaces an Intrinsic Derived Reference is typically used. It is a straight line representing a surface generatrix and the related tolerance is the straightness tolerance (ISO/TS 127801:2003). This tolerance is adequate to the nominal concept of ruled surface, but is difficult to verify practically. The method here proposed to verify the property of a surface to be ruled evaluates the uniformity of differential geometric properties along a straight line (a principal curvature is always null). In other words, a ruled surface in the neighbourhood of a generatrix can be approximated by a ruled paraboloid (figure $3)$.

With this approach the conformity of the generatrix to a straight line is not directly verified, but the uniformity of some local properties related to curvatures is (the neighborhood of the generatrix can be approximated by a unique ruled paraboloid). In what follows, the deviation from this kind of intrinsic reference is referred to as ruledness error. This approach to evaluate errors in ruled surfaces is possible thanks to the specific way to acquire the object under verification (which guarantees a high-density point cloud). It solves some problems dealing with the nature of the measurement process, which provides points that, in general, are not aligned with the surface generatrices. Furthermore, the generatrix direction is not preventively known and must be sought by investigating surface differential geometric properties. Hence, any straightness tolerance evaluation can be largely affected by mesh sizes. The high density of point clouds makes it possible to implement easily methods which perform local surface approximations. This method, when applied to tessellated surfaces, is a more rigorous way to evaluate ruledness and is intrinsically more robust than generatrices straightness tolerance verification. 


\section{Intrinsic nominal reference association}

In the GPS language the association corresponds to the feature operation: "fit an ideal feature to a non ideal feature according to a criterion" (ISO/TS 17450-1: 2005). In this work the criterion is referred to as rule and the association is carried out in order to quantitatively identify the nominal reference from which the error can be evaluated. The recognition step just performs a qualitative identification of the reference (analytical ideal feature), which must be evaluated quantitatively by approximating the non-ideal feature. For this purpose, several rules can be used, each of which identifies a different Intrinsic Shape Reference, which is affected by the rule under examination. At present, GPS standards do not define univocally the rules to identify ideal features. In literature, in order to carry out this operation, several rules are presented. Roughly speaking, the rules perform the median reference feature identification around which the tolerance zone is constructed. From a mathematical point of view, a constrained optimization problem needs to be solved. In order to determine the nominal reference $S$ (the ideal feature) that best fits the related point cloud $\mathbf{p}_{\mathbf{i}}$ (non-ideal feature), the most used association rule is based on the $\mathrm{L}_{\alpha}$ - norm. It is defined as follows:

$$
\mathrm{L}_{\alpha}=\left[\frac{1}{\mathrm{~N}} \sum_{\mathrm{i}=1}^{\mathrm{N}}\left|\mathrm{d}\left(\mathrm{S}, \mathbf{p}_{\mathbf{i}}\right)\right|^{\alpha}\right]^{1 / \alpha}
$$

where $0<\alpha<\infty, N$ is the total number of data points and $d\left(S, \mathbf{p}_{\mathbf{i}}\right)$ is the shortest distance (or equivalently, the residual error) between $\mathbf{p}_{\mathbf{i}}$ and $\mathbf{S}$. Based on the $\alpha$ value, several association rules are possible: the most used are summarized in table 2 .

From a practical point of view, the $\mathrm{L}_{1}$-norm and the $\mathrm{L}_{2}$-norm rules do not yield very different results. The $\mathrm{L}_{1}$-norm rule is less sensitive to outliers but can nevertheless offer multiple solutions. The $\mathrm{L}_{2}$-norm rule, on its part, can overestimate the error (especially, if we compare it to the error evaluated by the min - max approach), but it undoubtedly gives the best estimation of the nominal reference. With the $\mathrm{L}_{2}$-norm rule, the error can also be evaluated by following a statistical approach, based on the standard deviation of the points' distance from the estimated reference.

Table 2. The most used association rules for median reference feature evaluation

\begin{tabular}{|c|c|l|}
\hline & Rule name & RULE EXPRESSION \\
\hline $\boldsymbol{\alpha}=\mathbf{1}$ & $\mathbf{L}_{\mathbf{1}}$-norm rule & $\sum_{\mathrm{i}=1}^{\mathrm{N}}\left|\mathrm{d}\left(\mathrm{S}, \mathbf{p}_{\mathbf{i}}\right)\right|$ \\
\hline $\boldsymbol{\alpha}=\mathbf{2}$ & $\begin{array}{c}\mathbf{L}_{2} \text {-norm rule or } \\
\text { least squares approach }\end{array}$ & $\sum_{\mathrm{i}=1}^{\mathrm{N}} \mathrm{d}^{2}\left(\mathrm{~S}, \mathbf{p}_{\mathbf{i}}\right)$ \\
\hline $\boldsymbol{\alpha}=\infty$ & $\begin{array}{c}\mathbf{L}_{\boldsymbol{\alpha}} \text {-norm rule or } \\
\text { min }- \text { max approach }\end{array}$ & $\operatorname{Max}_{\mathrm{i}=1, \ldots \mathrm{N}}\left\{\mathrm{d}\left(S, \mathrm{p}_{\mathrm{i}}\right) \mid\right\}$ \\
\hline
\end{tabular}

The $\mathrm{L}_{\infty}$-norm rule (min - max approach) minimizes the maximum distance between the point $\mathbf{p}_{\mathbf{i}}$ and the ideal feature. In other words, the $\mathrm{L}_{\infty}$-norm rule minimizes the following function:

$$
f=\max \left\{d_{i}\right\}-\min \left\{d_{i}\right\}
$$




\subsection{The Intrinsic Shape Reference association}

The quantitative definition of the Intrinsic Shape Reference involves, respectively, the evaluation and the identification of the corresponding intrinsic characteristics and/or situation features. In table 3 the intrinsic characteristics and the situation features for each ISR are reported. The intrinsic references, with the value of their characteristics and the location of the situation features, strictly depend on the rules used to evaluate them. For each type of $I S R$, the $\mathrm{L}_{2}$-norm and the $\mathrm{L}_{\infty}$-norm rules have been implemented.

Table 3. The intrinsic characteristics and the situation features of the Intrinsic Shape References

\begin{tabular}{l|l|c} 
Intrinsic Shape Reference & Intrinsic characteristics & Situation features \\
\hline Plane & none & $\begin{array}{l}\bullet \quad \text { a point } \mathbf{x}_{\mathbf{0}} \equiv\left(\mathrm{x}_{0}, \mathrm{y}_{0}, \mathrm{z}_{0}\right) \text { located on the plane; } \\
\text { the direction } \mathbf{a} \equiv(\mathrm{a}, \mathrm{b}, \mathrm{c}) \text { of the plane; }\end{array}$ \\
\hline Sphere & diameter & $\bullet \quad$ its centre $\mathbf{x}_{\mathbf{0}} \equiv\left(x_{0}, y_{0}, z_{0}\right)$
\end{tabular}

\subsection{The Intrinsic Derived Reference association: the case of the axially - symmetric features}

Not every Intrinsic Derived Reference can be directly deduced from the point cloud. Some of them need a characteristic situation reference to be preventively estimated. This is also the case of the axially-symmetric non-ideal feature.

The quality of its estimation affects significantly the subsequent error evaluation. In this work, in order to estimate the axis, two methods are implemented: the normal intersections method (Pottmann et al. 1999) (henceforth denoted as $\Pi_{1}$ ) and the curvature centres method (Cao and Mumford 2002) (from now on denoted as $\left.\Pi_{2}\right)$. These rules must be stated in the tolerance specification. The axis itself is an Intrinsic Derived Reference, but it is also used to identify the set of cutting planes (perpendicular to the axis), from which the circularity and the location of the centre of the section ("associated derived centres" (UNI EN ISO 14660-2: 2002)) can be evaluated. The non-ideal circumferential line is extracted by projecting the points in the neighbourhood of the cutting plane onto it. Based on the optimization criterion, 
which is defined by a specific rule, for each plane section a reference ideal circle is associated with the non-ideal circumferential line. In this work the ideal circle is obtained by using the $\mathrm{L}_{2}$-norm and the $\mathrm{L}_{\infty}$-norm rules.

\subsection{The Intrinsic Local Reference association}

In order to evaluate profile regularity, surface regularity and surface ruledness, Intrinsic Local References need to be conveniently defined and associated. The rules here proposed to identify these nominal references can be considered as examples. Other rules could be defined to evaluate new error categories that require Intrinsic Local References. Since these rules play an important role in reference identification, error specification must also define the way to evaluate these intrinsic references.

\section{Profile regularity}

Roughly speaking, a regular profile is assimilable to a differentiable curve and any imperfection is associated with a deviation from it. This work takes the best local approximation of the data point with a regular curve as the nominal reference for profile regularity evaluation. The rules to identify the nominal reference include: the type of regular curve (polynomial curve, exponential curve, etc.) and the method to locally approximate the profile. In the approach herein proposed, the reference curve is evaluated, at each point of the profile, as the approximating curve of its neighbourhood. It is a quadric or a cubic polynomial, calculated by the weighted $\mathrm{L}_{2}$ norm rule. The weights of the approximation rule are assumed as the values of a Gaussian function having the mean located at the analyzed point and a properly selected value for the standard deviation $\sigma$ (figure 4). This weighting approach aims at defining a regular profile by filtering the local irregularities. The width of this filter is conventionally assumed as $\lambda=6 \sigma$ of the Gaussian function. The $\lambda$ value is assumed as the maximum value between the expected maximum size of the profile imperfection and the mesh dimension. The profile regularity error, at each point analyzed, is defined as the distance between the point and the related approximating curve.

\section{Surface regularity}

Surface regularity is a generalization (three - dimensional extension) of the profile regularity concept. A non-ideal feature consists of adjacent mesh vertices having some uniform differential geometric properties. Surface regularity measures locally the membership of each point to a regular surface which locally approximates its neighbourhood. Similarly to profile regularity, the rules to identify the nominal reference include: the type of regular surface and the method to locally approximate the surface. For each recognized non-ideal feature, the reference surface is evaluated at each vertex as the approximating surface by means of the weighted $\mathrm{L}_{2}$-norm rule. Surface regularity is not related to a specific shape of surface; it refers to the differentiability, which is a local property. Thus, in this work, the quadric paraboloid is used as a reference; it is the typical analytical regular surface used to evaluate the differential geometric properties. The weights of the approximation rule are assumed as the values of a two - dimensional symmetric Gaussian function having the mean located at the analyzed point and a properly selected value for the standard deviation 


\section{Error evaluation}

The final phase of the methodology herein proposed is error evaluation. For each type of non-ideal feature (see table 4), specific categories of errors can be 
identified. These are the categories that can be automatically evaluated as deviations from the recognizable intrinsic references. Table 4 does not show the curve and the surface profile tolerances, which rather require that a nominal reference be specified.

\begin{tabular}{|c|c|c|c|c|c|c|c|c|c|c|}
\hline & \multicolumn{8}{|c|}{$\begin{array}{c}\text { 1 able 4. rypes of non-1deal teature and related errors } \\
\text { Type of feature }\end{array}$} \\
\hline & & & \multirow{2}{*}{ plane } & \multirow{2}{*}{$\begin{array}{c}\text { sphere } \\
--\end{array}$} & \multirow{2}{*}{$\begin{array}{c}\text { cylinder } \\
\text { Extracted } \\
\text { median } \\
\text { line }\end{array}$} & \multirow{2}{*}{$\begin{array}{c}\text { cone } \\
\text { Extracted } \\
\text { median line }\end{array}$} & \multirow{2}{*}{$\begin{array}{c}\begin{array}{c}\text { generic } \\
\text { axially- } \\
\text { symmetric }\end{array} \\
\begin{array}{c}\text { Extracted } \\
\text { median line }\end{array}\end{array}$} & \multirow{2}{*}{$\begin{array}{c}\begin{array}{c}\text { generic } \\
\text { extruded }\end{array} \\
--\end{array}$} & \multirow{2}{*}{$\begin{array}{c}\begin{array}{c}\text { generic } \\
\text { cone }\end{array} \\
--\end{array}$} & \multirow{2}{*}{ 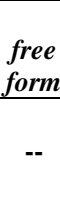 } \\
\hline \multirow{7}{*}{ 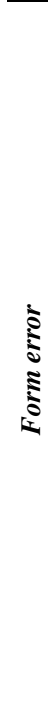 } & Straightness & - & & & & & & & & \\
\hline & Flatness & $\square$ & $\mathrm{X}$ & -- & -- & -- & -- & -- & -- & -- \\
\hline & Roundness & & -- & -- & $\begin{array}{c}\text { Any } \\
\text { extracted } \\
\text { cross- } \\
\text { sectional } \\
\text { circumfere } \\
\text { ntial line }\end{array}$ & $\begin{array}{c}\text { Any } \\
\text { extracted } \\
\text { cross- } \\
\text { sectional } \\
\text { circumferen } \\
\text { tial line }\end{array}$ & $\begin{array}{c}\text { Any extracted } \\
\text { cross-sectional } \\
\text { circumferentia } \\
1 \text { line }\end{array}$ & -- & -- & -- \\
\hline & $\begin{array}{c}\text { Total } \\
\text { Roundness }\end{array}$ & & -- & - & $\mathrm{X}$ & $\mathrm{X}$ & $\mathrm{X}$ & -- & -- & -- \\
\hline & Cylindricity & & -- & -- & $\mathrm{X}$ & -- & -- & -- & -- & -- \\
\hline & Conicity & $1 /$ & -- & -- & -- & $\mathrm{X}$ & -- & -- & -- & -- \\
\hline & Sphericity & $\theta$ & $\mathrm{X}$ & -- & -- & -- & -- & -- & -- & -- \\
\hline \multirow{2}{*}{ 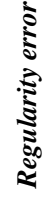 } & Profile & $r$ & $X$ & $\mathrm{X}$ & $\mathrm{X}$ & $\mathrm{X}$ & $\mathrm{X}$ & $\mathrm{X}$ & -- & -- \\
\hline & Surface & Ay & -- & -- & $\mathrm{X}$ & $\mathrm{X}$ & $\mathrm{X}$ & $\mathrm{X}$ & $\mathrm{X}$ & $\mathrm{X}$ \\
\hline \multicolumn{2}{|c|}{ Ruledness error } & & -- & -- & $\mathrm{X}$ & $\mathrm{X}$ & -- & $\mathrm{X}$ & $\mathrm{X}$ & -- \\
\hline
\end{tabular}

All the form errors can be expressed both as the maximum (Max) and as the standard deviation $(\sigma)$ of the point cloud distances from the corresponding intrinsic reference. A probabilistic evaluation of the error can be more significant than the maximum deviation measure. The point cloud acquisition process is affected by singular errors which can be ascribed to the typical measuring errors affecting the optical scanner devices. The $\sigma$ value performs a probabilistic estimation of the acquired point location with respect to the intrinsic nominal reference. This way to specify error is possible thanks to the large set of points acquired for each non-ideal feature.

\subsection{Error Evaluation: the case of the axially-symmetric features}

The typical form errors considered in the ISO standards for axially symmetric surfaces are roundness and axis straightness. For all these tolerances the Intrinsic Derived Reference is the axis of the feature, which is estimated by using specific rules such as those presented in section 6.2. Roundness error evaluation requires that, in any cross-section of the corresponding non-ideal feature (cylinder, 
cone and generic axially-symmetric), the non-ideal circumferential line be extracted (figure $8 \mathrm{~b}$ ). This line is compared to the circularity ideal reference and the error is calculated as the maximum radial distance between them. Axis straightness error evaluation requires the extraction of the median line of axially - symmetric features (cylindrical, conical and generic). The median line is the locus of the centres of the ideal circles associated with the non-ideal circumferential lines; in the GPS standards it is referred to as "associated derived centres" (UNI EN ISO 14660-2: 2002) (figure 8c). The median line is compared to the reference axis and the error is calculated as two times its maximum distance from the centres.

Thanks to the new techniques for geometric property measurement, which allow a large amount of point data to be automatically computed and virtual references to be simulated, new categories of tolerances can be introduced. This paper introduces the tolerance category of total roundness, suited to specify the maximum deviation from a nominal axially - symmetric surface. Traditional roundness performs single evaluations of the error, section by section (figure 8d). The total roundness error is similar to the run-out, but it is measured by virtually rotating the surface around its own estimated axis. Whereas the run-out requires that an external datum (axis) be assigned, total roundness is estimated from its intrinsic datum: its axis (its revolution axis). In this case, the intrinsic nominal references consist of a set of circles, all having their centre on the estimated axis (figure 8e). The algorithm used in this work for the automatic evaluation of the total roundness error is as follows:

(1) identification of the reference axis (rules $\Pi_{1}$ and $\Pi_{2}$ );

(2) definition of a cylindrical coordinate system $(\rho, \theta, \zeta)$ with $\zeta$ - axis coincident with the reference axis;

(3) circular projection (along the $\theta$ direction) of the point cloud onto a plane passing through the reference axis;

(4) evaluation of the local radial thickness of the projected point cloud.

\section{Application example}

The methodology, described in the previous sections, has been implemented in an original software, coded in $\mathrm{C}++$, by using a library dedicated to the processing of tessellated geometric models, which has been developed in the University of L'Aquila.

In order to verify the reliability of the proposed methodology, a specific test case, including different types of non-ideal features, has been analyzed. This test case (figure 9) refers to a real object whose acquisition has been carried out by an optical 3D scanner (www.scansystems.it). This test case consists of five non-ideal features, four of which are analytical features. Figure 10 shows the identification of features with their respective labels. The areas recognized as non-regular are depicted in yellow and therefore automatically excluded from the error evaluation. The results of the error analysis are quoted in table 5.

The rules used to estimate the intrinsic references play an important role in error evaluation. This is confirmed by the results reported in table 5, where it is possible to see how the different rules used give rise to differences in the estimated errors. For example, in the analysis of generic_axially-symmetric_1, different values for profile regularity errors are obtained by varying the rules for axis estimation $\left(\Pi_{1}\right.$ and $\Pi_{2}$ ) and for curve approximation (quadric and cubic polynomial). 


\section{Conclusion}

This paper proposes a general approach to form error evaluation and specification. This approach is founded on the concepts of non-ideal feature and intrinsic nominal reference. The object to be inspected is segmented into a set of nonideal features and for each of them one or more intrinsic nominal references are identified. The recognizability of non-ideal features and the rules to identify the intrinsic references from a point cloud are the key elements of this approach to tolerance specification. The tolerance categories that can be specified are those referring to recognizable non-ideal features for which intrinsic nominal references can be identified. Recognizable non-ideal features, with their intrinsic references, define the signifiers of the signs in a language specifically oriented to form error specification. These signs are suited to be automatically recognized in the inspected object. Based on the above-mentioned considerations, new error categories have been introduced. Some of these errors have to do with the intrinsic references deduced through the recognized differential geometric properties. 


\section{References}

Campana, F., Germani, M., 2008. Datum Identification for Tolerances Control on Dense Clouds of Points. Computer-Aided Design \& Applications, 5 (1-4), 209219.

Cao, Y., Mumford, D., 2002. Geometric Structure Estimation of Axially Symmetric Pots from Small Fragments. In Proceedings of SHAPE Lab. @SPPRA'02 Signal Processing, Pattern Recognition and Applications IASTED International Conference.

Di Angelo, L., Di Stefano, P., Morabito, A., 2007. Fuzzy sets for geometric shape recognition in triangular meshes. In Proceedings of the 6th International Conference on Intelligent Processing and Manufacturing of Materials (IPMM), Salerno, Italy, $25 \div 29$ June 2007 .

Di Angelo, L., Di Stefano, P., 2009. A New Method For Automatic Detection Of Non-Regular Points In Triangular Meshes. In Proceedings of XXI INGEGRAF - XVII ADM International Conference, Lugo, Spain, $10 \div 12$ June 2009.

Do Carmo, M., 1974. Differential Geometry of Curves and Surfaces, Prentice-Hall, Englewood Cliffs, N J.

Gao, J., Gindy, N., Chen, X., 2006. An automated GD\&T inspection system based on non-contact 3D digitization. International Journal of Production Research, 44 (1), 117-134.

Huang, J., 1999. An exact solution for the roundness evaluation problems. Precision Engineering, 23 (11), 2-8. 
ISO 1101: 2004, 2004. Geometrical Product Specifications (GPS) - Tolerances of form, orientation, location and run out.

UNI EN ISO 14660-2, 2002. Geometrical Product Specifications (GPS) -Geometrical features -- Part 2: Extracted median line of a cylinder and a cone, extracted median surface, local size of an extracted feature.

ISO/TS 12780-1:2003. Geometrical Product Specifications (GPS) -- Straightness -Part 1: Vocabulary and parameters of straightness.

ISO/TS 17450-1: 2005: "Geometrical product specification (GPS) - General concept - Part 1: Model for geometric specification and verification".

Lagarias, J. C., Reeds, J. A., Wright, M. H., Wright P. E., 1998. Convergence properties of the Nelder-Mead Simplex Method in low dimensions. Journal of Optimization, 9 (1), 112-147.

Li, Y. and Gu, P., 2004. Free-form surface inspection techniques - state of the art review. Computer-Aided Design, 36 (13), 1395-1417.

Li, Y. and Gu, P., 2005. Sculptured surface tolerance verification with design datums. International Journal of Production Research, 43 (7), 1465-1482.

Li, Y. and Gu, P., 2005. Inspection of free-form shaped parts. Robotics and Computer Integrated Manufacturing, 21 (4 - 5), 421-430.

Liu, Y. and Xiong, Y., 2008. Automatic segmentation of unorganized noisy point clouds based on the Gaussian map. Computer-Aided Design, 40 (5), 576-594.

Moroni, G, Petro, S., 2008. Geometric Tolerance Evaluation: a Discussion on Minimum Zone Fitting Algorithms. Precision Engineering, 32 (3), 232-237.

Nielsen, H. S., 2006. New Concepts in Specifications, Operators and Uncertainties and Their Impact on Measurement and Instrumentation. Measurement Science and Technology, 17 (3), 541-544.

Novaksy O., Barczak ALC., 1997. Utilization of Voronoi Diagrams for circularity algorithms. Manufacturing Review, 20 (3), 188-195.

Pottmann, H., Peternell, M., Rafani, B., 1999. An introduction to line geometry with applications. Computer-Aided Design, 31 (1), 3-16.

Prieto, F., Redarce, T., Lepage R., Boulanger, P., 2002. An Automated Inspection System. International Journal of Advanced Manufactured Technology, 19 (12), 917-925.

Traband, M.T., Joshi, S., Wysk, R.A., Cavalier, T.M., 1989. Evaluation of straightness and flatness tolerances using the minimum zone. Manufacturing Review, 2 (3), 189-195. 


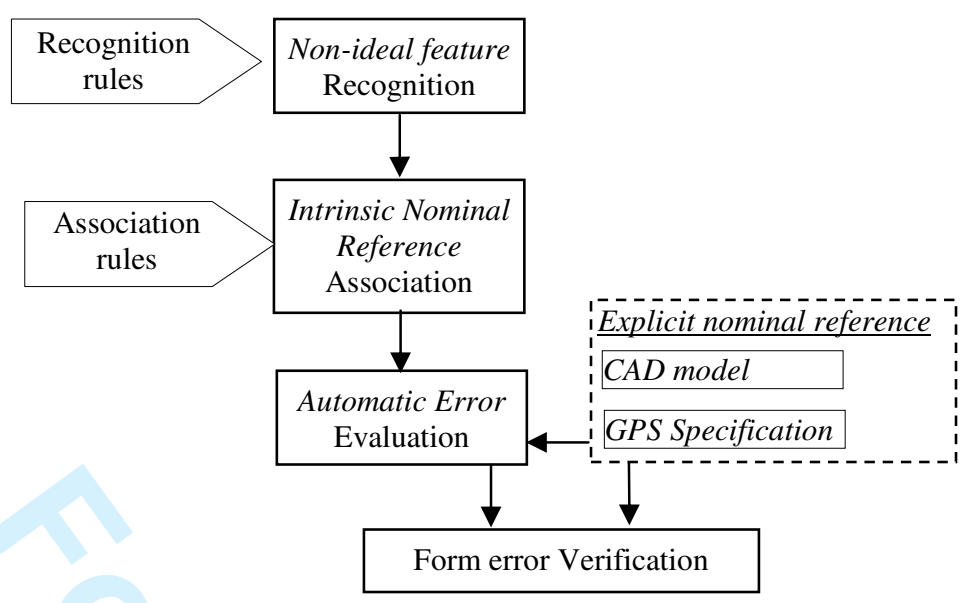

Figure 1. An approach to the automatic geometric inspection based on the recognition of the intrinsic references

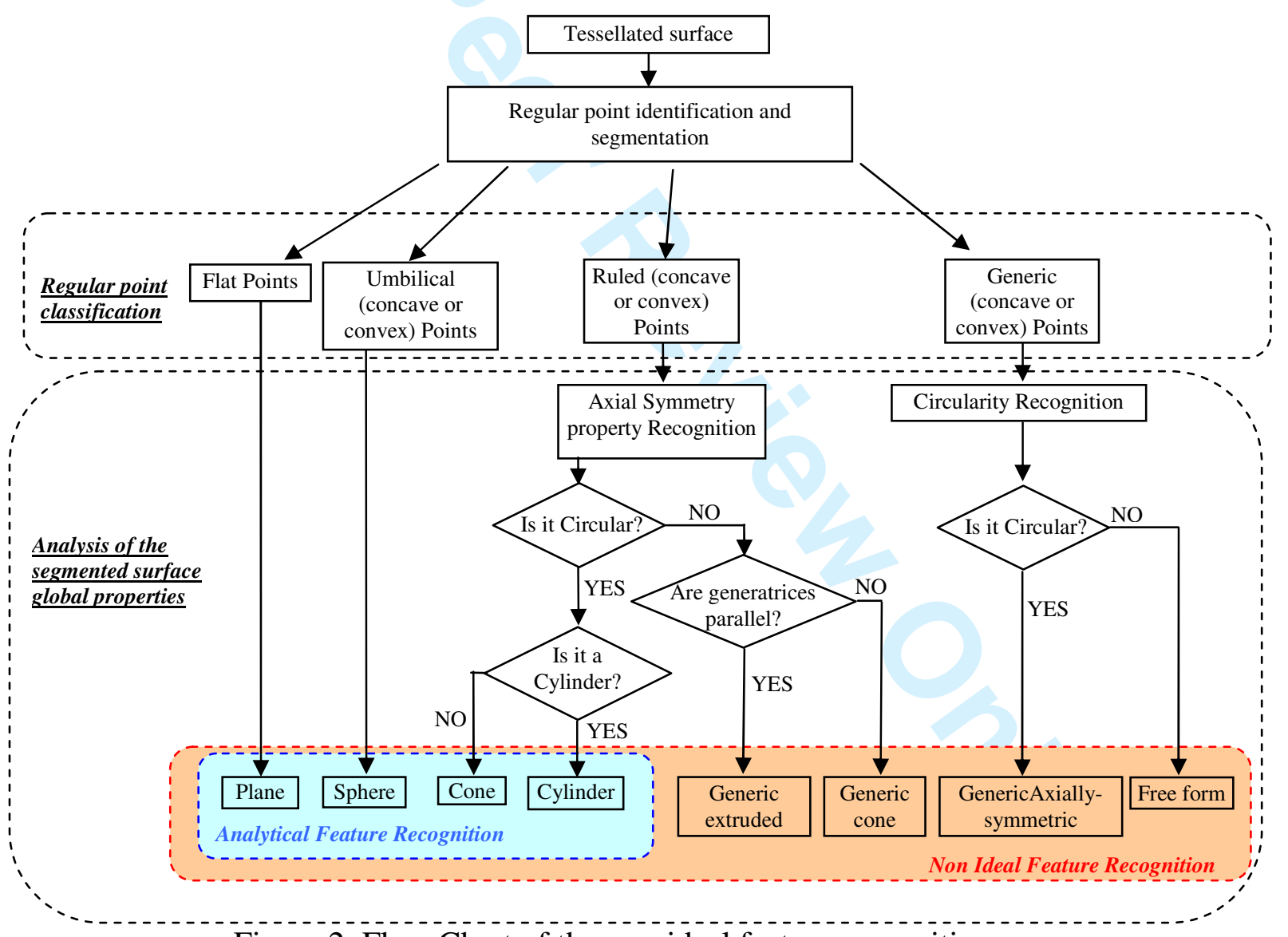

Figure 2. Flow Chart of the non-ideal feature recognition process 


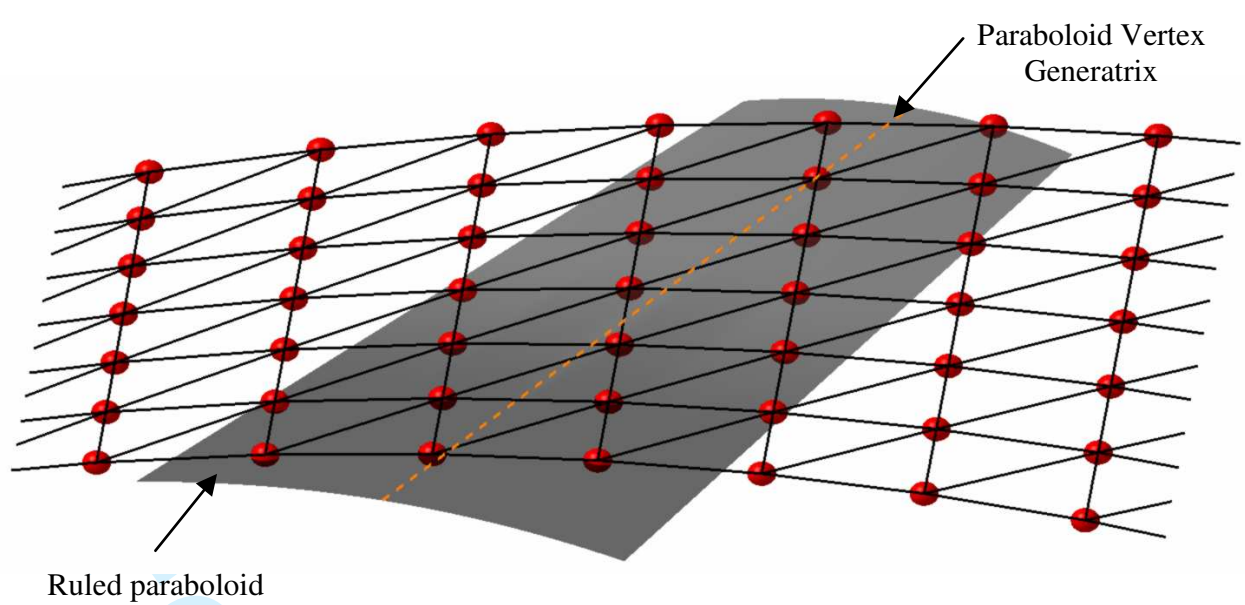

Figure 3. Approximation by a ruled paraboloid of the generatrix neighbourhood

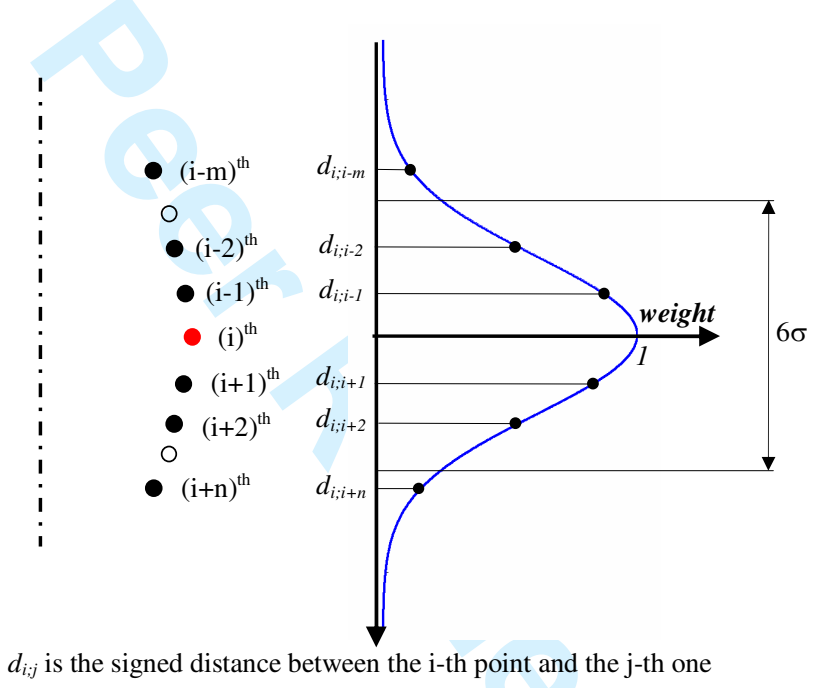

Figure 4. The weighting approach to define profile regularity: points on the profile with the superimposed weights function for curve approximation

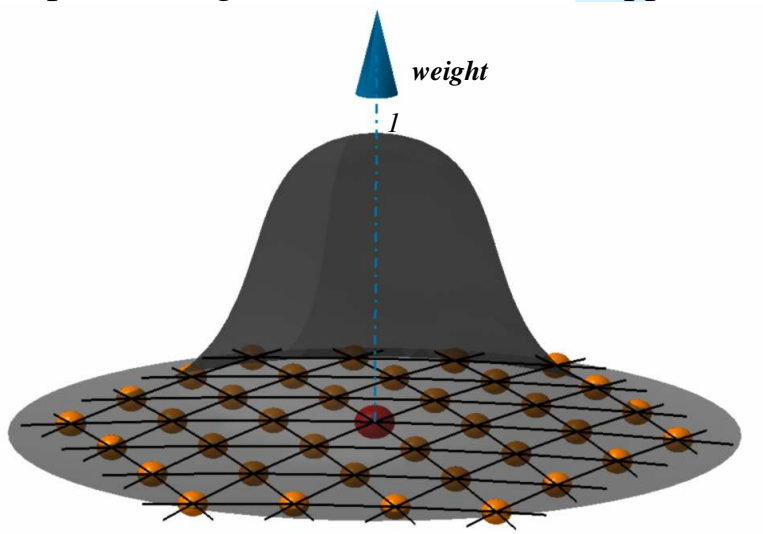

Figure 5. The weighting approach to define surface regularity: the point $p$ (in red) and its neighborhood (in orange) with the superimposed weighting function 


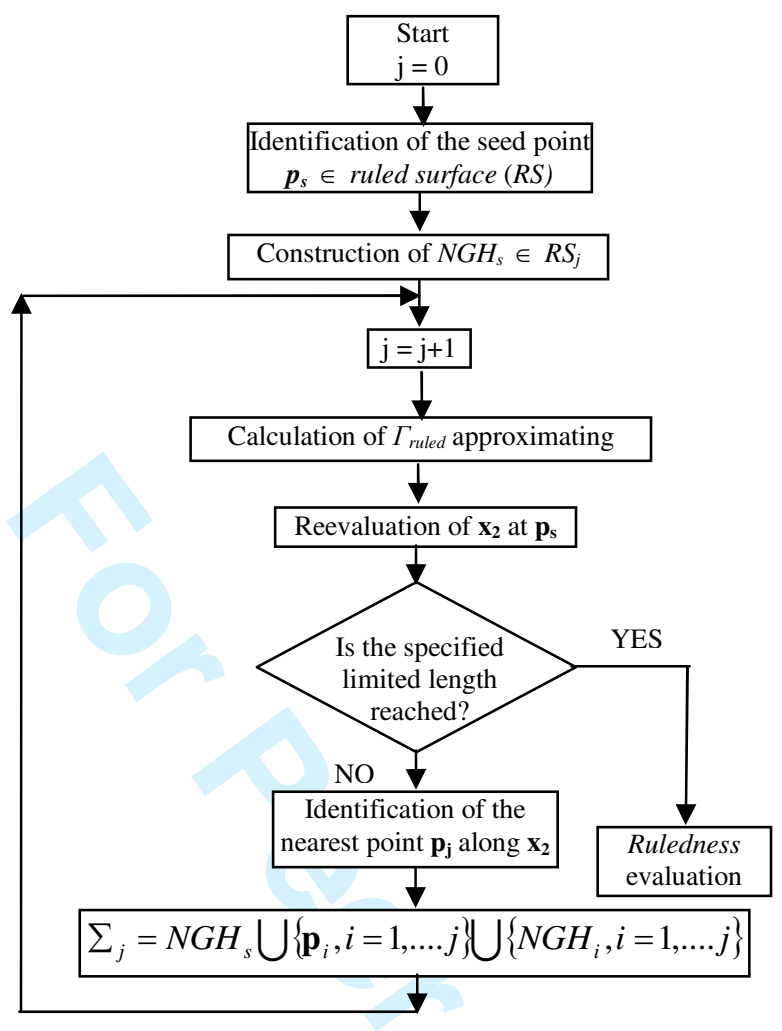

Figure 6. Flow Chart of the ruledness evaluation process
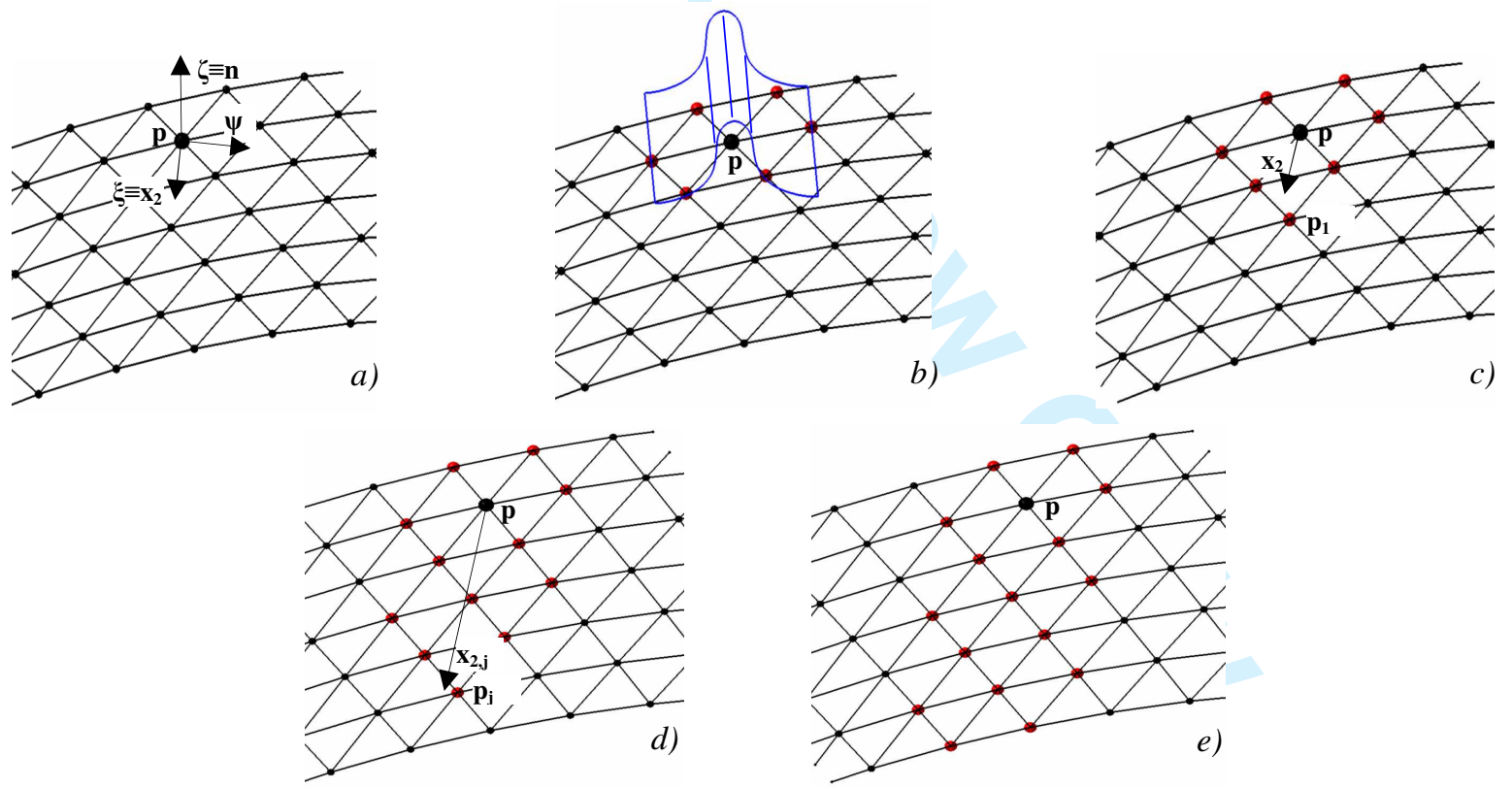

Figure 7. Explanation of the steps in the ruledness evaluation process 

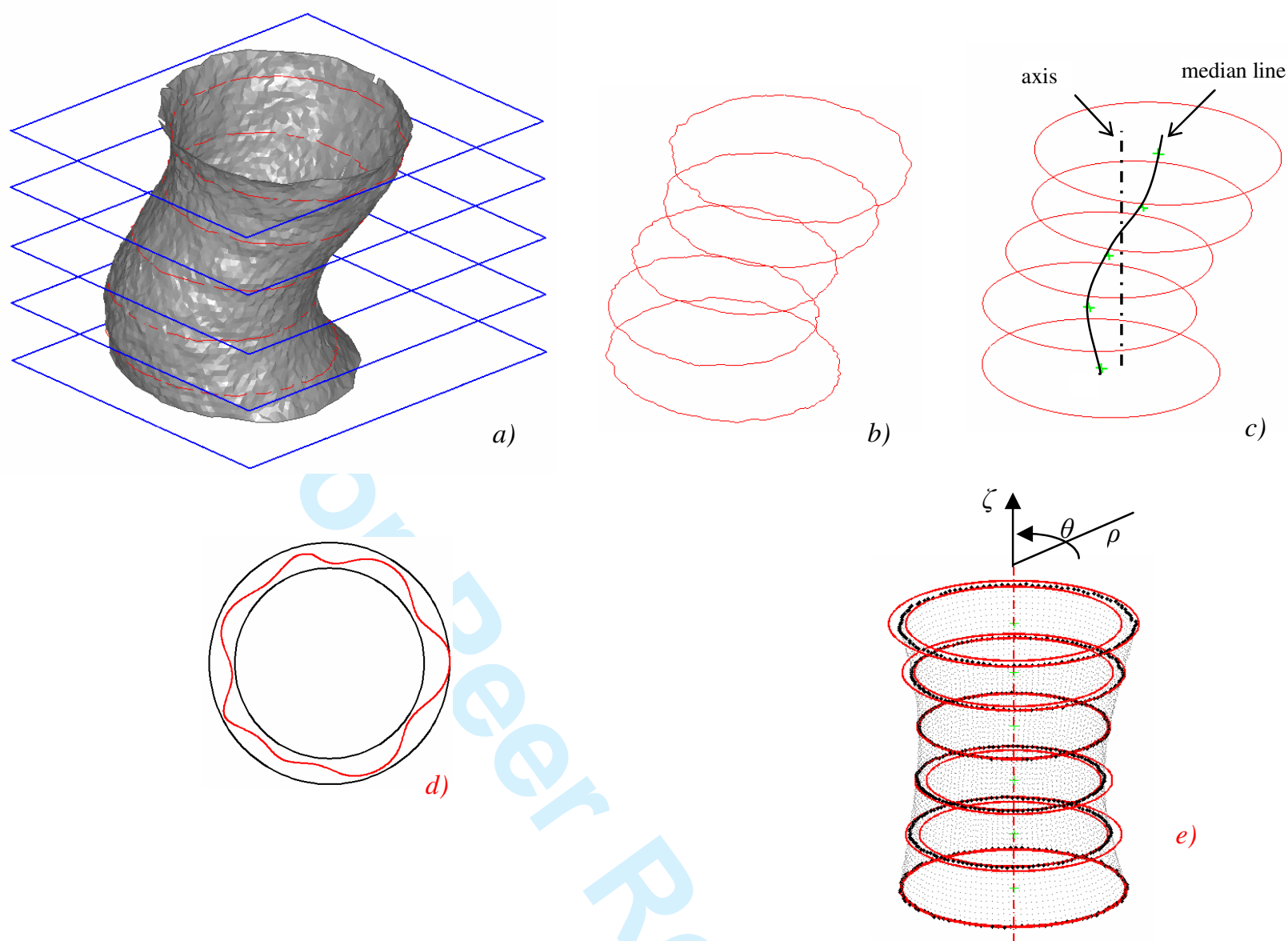

e)

Figure 8. Form error explanation

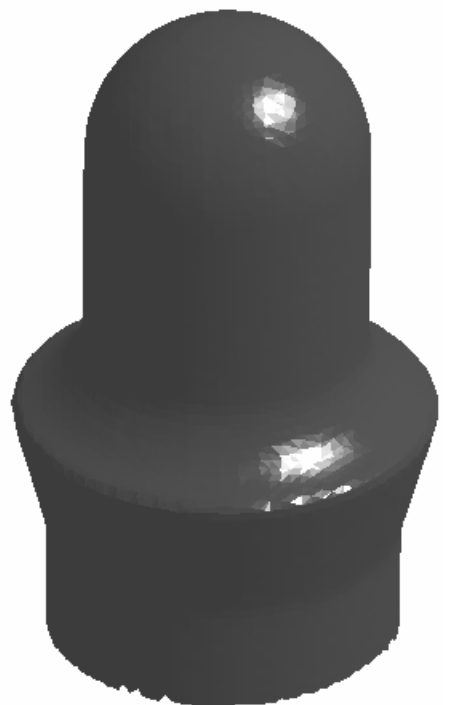

Figure 9. The test case considered $($ mesh dimension $=0.25 \mathrm{~mm})$

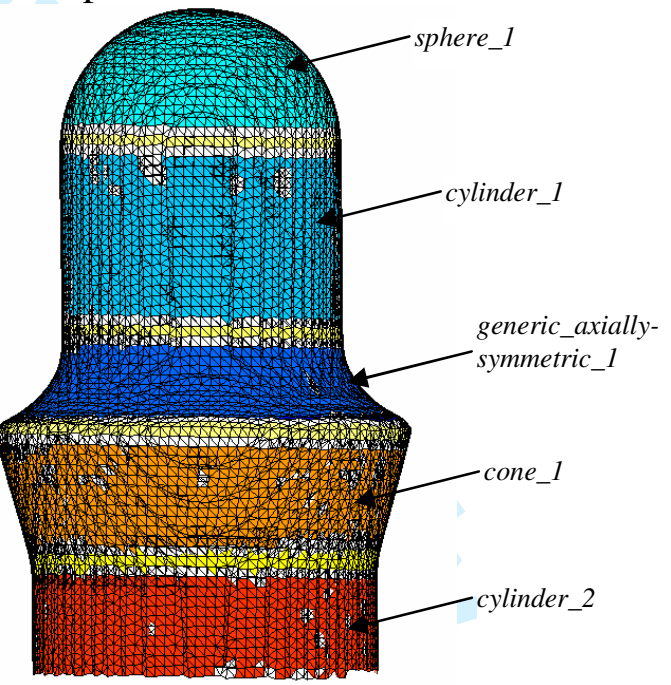

Figure 10. The result of the features' identification with the respective labels 
Figure 11. A screen capture of the our implementation system

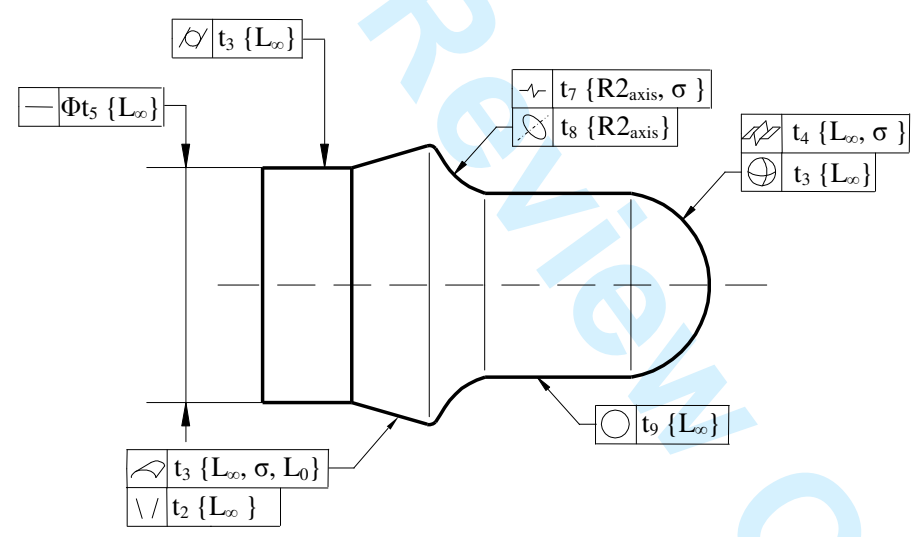

Figure 12. Examples of tolerance prescriptions applied to the drafting of the test case under analysis 